\title{
17-(2',5'-二取代噁唑基)-雄甾-4,16-二烯-3-酮的合成及抗肿瘤活性研究
}

\author{
黎龙香 ${ }^{a}$ 谢龙勇 ${ }^{a}$ 王风君 ${ }^{b}$ 何卫民*,a 向建南*,a \\ ( ${ }^{a}$ 湖南大学化学化工学院 长沙 410082) \\ ( ${ }^{b}$ 中南大学湘雅医院耳鼻喉科 长沙 410082)
}

\begin{abstract}
摘要 以 4-雄烯二酮 1 为原料, 用金催化甾炔法设计并合成了一系列 17-(2', $5^{\prime}$ 二二取代噁唑基)-雄甾-4,16-二烯-3-酮衍生 物 $4 \mathbf{a} \sim 4 \mathbf{k}$. 所合成产物通过 ${ }^{1} \mathrm{H}$ NMR, ${ }^{13} \mathrm{C}$ NMR, IR 和 HRMS 方法进行了结构表征. 以阿比特龙为阳性对照, 通过 3-(4,5-二甲基噻唑-2)-2,5-二苯基四氮唑溴盐(MTT)法测试了目标化合物对 MCF-7(人乳腺癌细胞)、A549(人肺癌细胞)、 Bel-7402(人肝癌细胞)、Hela (人宫颈癌细胞)和 PC-3M-1E8(人前列腺癌细胞)的体外抗肿瘤活性. 结果表明大多数化合 物表现出了较好的抗肿瘤活性, 其中化合物 $4 \mathrm{c}, 4 \mathrm{~g}, 4 \mathbf{i}$ 和 $4 \mathbf{j}$ 的抗肿瘤活性与阳性对照物阿比特龙相当，且所测化合物 对 MCF-7 有较好选择性作用, 其 $\mathrm{IC}_{50}$ 值在 3.0 25.5 $\mu \mathrm{mol} / \mathrm{L}$ 之间.
\end{abstract}

关键词 甾体; 噁唑; 金催化; 合成; 抗肿瘤活性

\section{Synthesis and Antitumor Activity of 17-(2',5'-Disubstituted-oxazolyl)-androsta-4,16-dien-3-one Deriva- tives}

\author{
Li, Longxiang $^{a} \quad$ Xie, Longyong $^{a} \quad$ Wang, Fengjun $^{b} \quad$ He, Weimin ${ }^{*, a}$ \\ Xiang, Jiannan*,a \\ ( ${ }^{a}$ College of Chemistry and Chemical Engineering, Hunan University, Changsha 410082) \\ ( ${ }^{b}$ Department of Otolaryngology-Head and Neck Surgery Xiangya Hospital, Central South University, Changsha 410082)
}

\begin{abstract}
A series of 17-(2',5'-disubstituted-oxazolyl)-androsta-4,16-dien-3-one derivatives $\mathbf{4 a} \sim \mathbf{4 k}$ were designed and synthesized via the gold-catalyzed steroidal alkyne oxidation from 4-androstene-3,17-dione 1 . The structures of the targeted compounds were characterized by ${ }^{1} \mathrm{H}$ NMR, ${ }^{13} \mathrm{C}$ NMR, IR and HRMS. These synthesized compounds were screened for in vitro antitumor activity by 3-(4,5-dimethyl-2-thiazolyl)-2,5-diphenyl-2H-tetrazolium bromide (MTT) assay against MCF-7 (human breast cancer cell line), A549 (human lung cancer cell line), Bel-7402 (human liver cancer cell line), Hela (human cervical cancer cell line) and PC-3M-1E8 (human prostate cancer cell line) using abiraterone as the positive control. The results revealed that most of compounds showed moderate to significant activity. In particular, compounds $\mathbf{4 c}, \mathbf{4 g}, \mathbf{4 i}$ and $\mathbf{4 j}$ exhibited similar antitumor activities compared with that of abiraterone, and all tested compounds displayed higher selectivity against MCF-7 cell line $\left(\mathrm{IC}_{50}=3.0 \sim 25.5 \mu \mathrm{mol} / \mathrm{L}\right)$.

Keywords steroids; oxazole; gold-catalyzed; synthesis; antitumor activity
\end{abstract}

甾体化合物广泛存在于自然界的动植物中 ${ }^{[1]}$, 在抗 癌 ${ }^{[2]}$ 、抗炎 ${ }^{[3]}$ 、心血管疾病 ${ }^{[4]}$ 以及免疫 ${ }^{[5]}$ 等方面都表现出 了潜在的药物效果. 通过对甾体化合物母体进行合理的 结构修饰, 可以明显提高甾体的生物活性 ${ }^{[6]}$. 最近几十 年来, 在甾体的 17-位引入杂环获得了巨大关注, 许多 甾体 17-位被引入杂环后对 17 $\alpha$-差弪化酶(P45017 $)$ )具有显
著的抑制作用. 例如，已经上市的商品抗前列腺癌药物 阿比特龙(Scheme 1) 以及甾体 17-位被引入杂环咪唑、异 噁唑、吡唑 ${ }^{[7]}$ 、三唑 ${ }^{[8]}$ 、噻唑、噁唑 ${ }^{[9] 、}$ 、哌嗪 ${ }^{[10]}$ 、苯基 吡唑 ${ }^{[11]}$ 等, 这些甾体 17-位连不同杂环对 P45017 $\alpha$ 表现 出了不同程度的抑制作用. 有些 17-位杂环甾体还表现

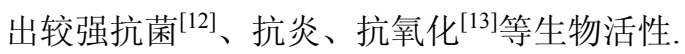

*E-mail: jnxiang@hnu.edu.cn

Received February 27, 2014; revised April 15, 2014; published online May 5, 2014.

Project supported by the National Natural Science Foundation of China (Nos. 21302048, 21276068), the China Postdoctoral Science Foundation (No. 2013M540625) and the Natural Science Foundation of Hunnan Province (No. 11JJ5008).

国家自然科学基金(Nos. 21302048, 21276068)、中国博士后科学基金(No. 2013M540625)、湖南省自然科学基金(No. 11JJ5008)资助项目. 


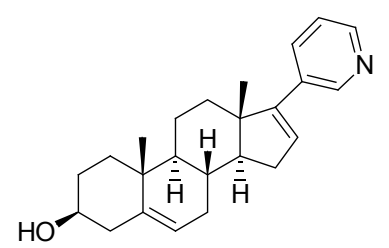

Scheme 1

噁唑是一类非常重要的含氧氮唑类杂环化合物, 它 不仅在有机化学中发挥着非常重要的作用, 还具有广泛 的生理活性 ${ }^{[14 \sim 16]}$. 噁唑化合物的合成可以由多官能团 的单组份化合物分子内环化得到 ${ }^{[17]}$, 也可以通过几种 含氮氧的简单化合物多组分反应得到 ${ }^{[18]}$. Zhang 等 ${ }^{[19]}$ 在 2011 年报道了一种通过金催化末端炔烃氧化形成的 $\alpha$ 羰基金卡宾中间体与腈反应生成 2,5-二取代噁唑的方 法, 腈在反应中既作为溶剂又作为反应物. 这种金催化 制备噁唑的方法反应条件温和、产率高、底物适用性好. 通过近几年我们对炔以及金催化的研究 ${ }^{[20,21]}$, 我们设想 能否利用 Zhang 发展的金催化方法在甾炔的 17-位引入 2,5-二取代啞唑杂环. 虽然已经有关于甾体 17-位连噁唑 杂环的文献报道, 但现有报道反应路线长、总产率低, 而且噁唑的取代位置大多数是在 2-位和 4-位上 ${ }^{[9]}, 2,5-$ 二取代啞唑杂环连在甾体 17-位还没有相关报道. 4-雄烯 二酮 1 是制备孕激素、皮质激素、雄激素和避孕药等甾 体药物的重要中间体, 我们拟以 4-雄烯二酮 1 为起始 原料, 通过乙炔的亲核加成在甾体 17-位引入炔基生成 甾炔醇 $\mathbf{2}$, 甾炔醇 $\mathbf{2}$ 脱羟基生成甾炔 $\mathbf{3}$, 甾炔 $\mathbf{3}$ 在金催化 下与不同腈反应合成了 17-(2',5'-二取代噁唑基)-雄甾4,16-二烯-3-酮衍生物 4a $\sim \mathbf{4 k}$, 并用 3-(4,5-二甲基噻唑2)-2,5-二苯基四氮唑溴盐(MTT)法对所合成的目标物进 行了体外抑制肿瘤细胞增殖活性测试.

\section{1 结果与讨论}

\section{1 合成}

本研究在 Zhang 等 ${ }^{[19]}$ 工作的基础上, 进一步优化反 应的条件. 我们选用化合物 3 和乙腈作为模型反应, 研 究了金催化剂、氧化剂、温度对反应的影响, 实验结果 见表 1. 首先, 本实验考察了金催化剂对反应的影响(表 1, Entries $1 \sim 8$ ), 实验发现, 在 $\mathrm{Ph}_{3} \mathrm{PAuNTf}_{2}$ 和 IP$\mathrm{rAuNTf}_{2}$ 的催化下, 核磁理论收率最佳(均为 $72 \%$ ). 考虑 到 $\mathrm{Ph}_{3} \mathrm{PAuNTf}_{2}$ 比 $\mathrm{IPrAuNTf}_{2}$ 廉价易得, 所以我们采用 $\mathrm{Ph}_{3} \mathrm{PAuNTf}_{2}$ 为最佳催化剂. 然后, 用 $\mathrm{Ph}_{3} \mathrm{PAuNTf}_{2}$ 作为 催化剂, 研究了氧化剂对反应的影响(表 1, Entries 1,9 11), 结果表明 8-甲基喹啉氮氧为氧化剂时核磁理论收 率最高达 $72 \%$. 最后, 考察了在最佳反应条件下温度的 影响(表 1, Entries 1, 12 14), 反应体系在室温、60,70
及 $90{ }^{\circ} \mathrm{C}$ 中的核磁理论产率差异较大，分别是 $48 \%$, $72 \%, 72 \%$ 和 $56 \%$. 因此，反应的最佳温度是 $60{ }^{\circ} \mathrm{C}$.

表 1 优化反应条件 ${ }^{a}$

Table 1 Screening of reaction conditions

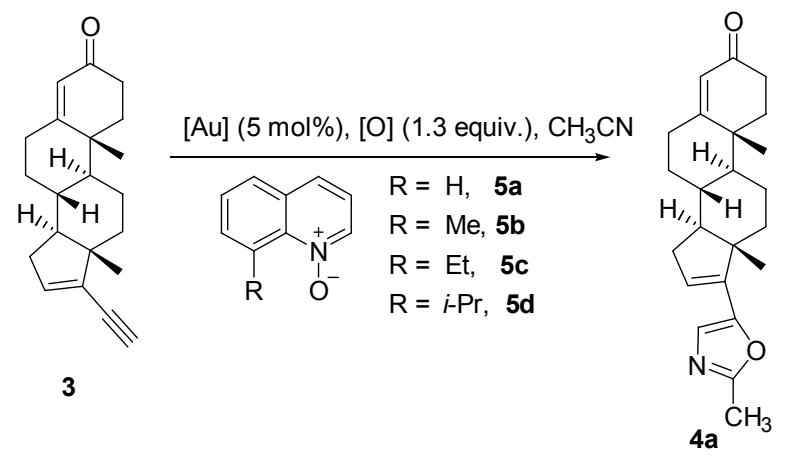

\begin{tabular}{clccc}
\hline Entry & \multicolumn{1}{c}{ Gold catalyst } & Oxidant & Conditions $^{b}$ & Yield $^{c} \%$ \\
\hline 1 & $\mathrm{Ph}_{3}$ PAuNTf $_{2}$ & $\mathbf{5 b}$ & $60{ }^{\circ} \mathrm{C}, 8 \mathrm{~h}$ & $72^{d}$ \\
2 & $\mathrm{Et}_{3} \mathrm{PAuNTf}_{2}$ & $\mathbf{5 b}$ & $60{ }^{\circ} \mathrm{C}, 8 \mathrm{~h}$ & 68 \\
3 & $\mathrm{Cy}_{3} \mathrm{PAuNTf}_{2}$ & $\mathbf{5 b}$ & $60{ }^{\circ} \mathrm{C}, 8 \mathrm{~h}$ & 69 \\
4 & IPrAuNTf $_{2}$ & $\mathbf{5 b}$ & $60{ }^{\circ} \mathrm{C}, 8 \mathrm{~h}$ & 72 \\
5 & BrettPhosAuNTf $_{2}$ & $\mathbf{5 b}$ & $60{ }^{\circ} \mathrm{C}, 8 \mathrm{~h}$ & 70 \\
6 & $\mathrm{Ph}_{3}{\mathrm{PAuCl} / \mathrm{AgNTf}_{2}}$ & $\mathbf{5 b}$ & $60{ }^{\circ} \mathrm{C}, 8 \mathrm{~h}$ & 56 \\
7 & $\mathrm{AgNTf}_{2}$ & $\mathbf{5 b}$ & $60{ }^{\circ} \mathrm{C}, 8 \mathrm{~h}$ & 0 \\
8 & $\mathrm{HNTf}_{2}$ & $\mathbf{5 b}$ & $60{ }^{\circ} \mathrm{C}, 8 \mathrm{~h}$ & 0 \\
9 & $\mathrm{Ph}_{3} \mathrm{PAuNTf}_{2}$ & $\mathbf{5 a}$ & $60{ }^{\circ} \mathrm{C}, 8 \mathrm{~h}$ & 54 \\
10 & $\mathrm{Ph}_{3} \mathrm{PAuNTf}_{2}$ & $\mathbf{5 c}$ & $60{ }^{\circ} \mathrm{C}, 8 \mathrm{~h}$ & 68 \\
11 & $\mathrm{Ph}_{3} \mathrm{PAuNTf}_{2}$ & $\mathbf{5 d}$ & $60{ }^{\circ} \mathrm{C}, 8 \mathrm{~h}$ & 63 \\
12 & $\mathrm{Ph}_{3} \mathrm{PAuNTf}_{2}$ & $\mathbf{5 b}$ & $\mathrm{r.t}, 12 \mathrm{~h}$ & 48 \\
13 & $\mathrm{Ph}_{3} \mathrm{PAuNTf}_{2}$ & $\mathbf{5 b}$ & $70{ }^{\circ} \mathrm{C}, 8 \mathrm{~h}$ & 72 \\
14 & $\mathrm{Ph}_{3} \mathrm{PAuNTf}_{2}$ & $\mathbf{5 b}$ & $90{ }^{\circ} \mathrm{C}, 8 \mathrm{~h}$ & 56 \\
\hline
\end{tabular}

${ }^{a}$ In vials; [3] $=0.05 \mathrm{~mol} / \mathrm{L} .{ }^{b}$ Monitored by TLC. ${ }^{c}$ NMR yield using diethyl phthalate as the internal reference. ${ }^{d}$ Isolated yield $70 \%$.

由表 1 可知, 甾炔 $\mathbf{3}$ 和腈的最佳反应条件: 催化剂 为 $5 \mathrm{~mol} \% \mathrm{Ph}_{3} \mathrm{PAuNTf}_{2}$, 氧化剂为 1.3 equiv. 的 8-甲基喹 啉氮氧, 反应温度为 $60{ }^{\circ} \mathrm{C}$, 反应时间为 $8 \mathrm{~h}$.

根据上述优化条件，目标化合物 $\mathbf{4 a} \sim \mathbf{4 k}$ 的合成路 线如 Scheme 2 所示. 化合物 $\mathbf{2}$ 和 $\mathbf{3}$ 通过参考文献[22, 23] 合成所得，随后甾炔 $\mathbf{3}$ 在金催化下与不同腈氧化制备得 到 $4 a \sim 4 k$. 在合成化合物 $4 a \sim 4 i$ 的过程中，腈既作反应 物又作反应溶剂; 对于由固体腈合成的化合物 $\mathbf{4 j}, \mathbf{4 k}$, 我们用惰性溶剂氯苯作溶剂. 所有合成的化合物都通过 ${ }^{1} \mathrm{H} N M R,{ }^{13} \mathrm{C}$ NMR, IR 和 HRMS 方法进行了结构表征.

\section{2 生物活性}

采用 MTT 法, 以人乳腺癌细胞(MCF-7)、人肺癌细 胞(A549)、人肝癌细胞(Bel-7402)、人宫颈癌细胞(Hela) 和人前列腺癌细胞(PC-3M-1E8)作为笁选对象, 分别对 化合物 3 和 $4 \mathrm{a} \sim 4 \mathrm{k}$ 进行了抗肿瘤活性篎选，结果见表 2.

如表 2 所示, 大部分化合物具有较好的体外抗肿瘤 

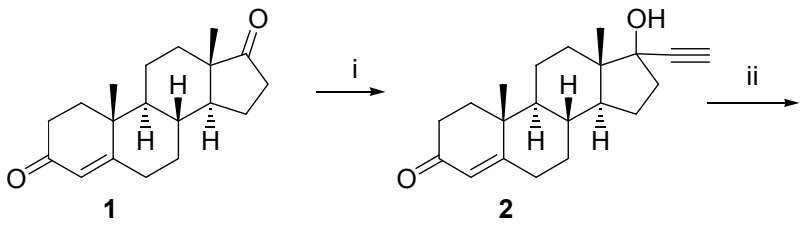

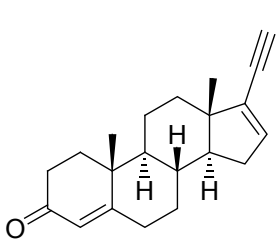

3

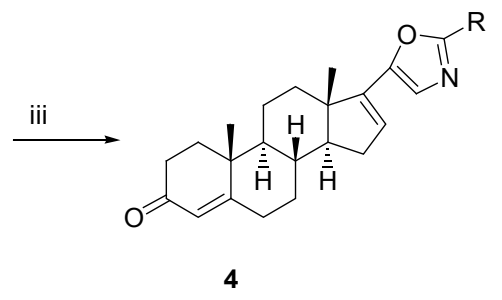

$$
\begin{aligned}
& \text { 4a } \mathrm{R}=\mathrm{CH}_{3} \text {, 4b R }=\mathrm{CH}_{2} \mathrm{CH}_{2} \mathrm{CH}_{3}, \mathbf{4 c} \mathrm{R}=\mathrm{CH}_{2}\left(\mathrm{CH}_{2}\right)_{2} \mathrm{CH}_{3} \text {, } \\
& \text { 4d R = } \mathrm{CH}_{2}\left(\mathrm{CH}_{2}\right)_{3} \mathrm{CN}, \mathbf{4 e} \mathrm{R}=\mathrm{CH}_{2} \mathrm{CH}_{2} \mathrm{CH}_{2} \mathrm{Cl} \text {, 4f } \mathrm{R}=\mathrm{CH}=\mathrm{CH}_{2} \text {, } \\
& \text { 4g R }=-\left\langle\text {, 4h R }=\mathrm{C}_{6} \mathrm{H}_{5}, \mathbf{4 j ~ R}=4-\mathrm{CH}_{3} \mathrm{C}_{6} \mathrm{H}_{4}, \mathbf{4 k ~ R}=2-\mathrm{BrC}_{6} \mathrm{H}_{4}\right. \text {, } \\
& \text { 4i } R=2-P y
\end{aligned}
$$

Reagents and conditions: (i) $t$-BuOK, THF, $\mathrm{C}_{2} \mathrm{H}_{2}$, r.t., $8 \mathrm{~h}$; (ii) imidazole, $\mathrm{SOCl}_{2}$, THF, $5 \sim 10{ }^{\circ} \mathrm{C}, 8 \mathrm{~h}$; (iii) 8-methylquinoline $\mathrm{N}$-oxide, $\mathrm{Ph}_{3} \mathrm{PAuNTf}_{2}, \mathrm{RCN}, 60{ }^{\circ} \mathrm{C}, 8 \mathrm{~h}$ or 8 -methylquinoline $\mathrm{N}$-oxide, $\mathrm{Ph}_{3} \mathrm{PAuNTf}_{2}, \mathrm{RCN}$, chlorobenzene, $60{ }^{\circ} \mathrm{C}, 8 \mathrm{~h}$

\section{Scheme 2}

表 2 目标化合物的体外抗肿瘤活性 $\left(\mathrm{IC}_{50}, \mu \mathrm{mol} / \mathrm{L}\right)$ Table 2 Antitumor activities of target compounds in vitro $\left(\mathrm{IC}_{50}\right.$, $\mu \mathrm{mol} / \mathrm{L})$

\begin{tabular}{cccccc}
\hline Compd. & MCF-7 & A549 & Bel-7402 & Hela & PC-3M-1E8 \\
\hline $\mathbf{3}$ & $>50$ & $>50$ & $>50$ & $>50$ & $>50$ \\
$\mathbf{4 a}$ & $12.6 \pm 0.4$ & $36.4 \pm 0.8$ & $42.2 \pm 0.7$ & $46.0 \pm 0.5$ & $48.2 \pm 0.6$ \\
$\mathbf{4 b}$ & $6.9 \pm 0.2$ & $18.6 \pm 0.7$ & $20.3 \pm 0.9$ & $23.1 \pm 0.4$ & $27.4 \pm 0.4$ \\
$\mathbf{4 c}$ & $3.6 \pm 0.2$ & $13.2 \pm 0.5$ & $10.8 \pm 0.6$ & $11.4 \pm 0.6$ & $12.8 \pm 0.5$ \\
$\mathbf{4 d}$ & $3.0 \pm 0.3$ & $14.4 \pm 0.4$ & $11.4 \pm 0.4$ & $13.9 \pm 0.5$ & $14.6 \pm 0.7$ \\
$\mathbf{4 e}$ & $5.5 \pm 0.5$ & $16.8 \pm 0.7$ & $14.7 \pm 0.5$ & $16.2 \pm 1.0$ & $17.8 \pm 0.9$ \\
$\mathbf{4 f}$ & $7.2 \pm 0.2$ & $25.2 \pm 0.5$ & $28.5 \pm 0.7$ & $25.5 \pm 0.8$ & $27.4 \pm 0.4$ \\
$\mathbf{4 g}$ & $6.9 \pm 0.3$ & $12.0 \pm 0.3$ & $10.2 \pm 0.7$ & $13.7 \pm 0.5$ & $9.3 \pm 0.5$ \\
$\mathbf{4 h}$ & $25.5 \pm 0.5$ & $>50$ & $>50$ & $>50$ & $>50$ \\
$\mathbf{4 i}$ & $3.8 \pm 0.2$ & $7.5 \pm 0.5$ & $8.4 \pm 0.8$ & $7.8 \pm 0.9$ & $12.1 \pm 0.6$ \\
$\mathbf{4 j}$ & $4.5 \pm 0.3$ & $9.3 \pm 0.8$ & $6.9 \pm 0.5$ & $11.1 \pm 0.4$ & $9.6 \pm 0.3$ \\
$\mathbf{4 k}$ & $9.0 \pm 0.4$ & $27.8 \pm 0.7$ & $19.2 \pm 0.6$ & $18.6 \pm 0.4$ & $20.1 \pm 0.7$ \\
阿比特 & $3.2 \pm 0.3$ & $11.2 \pm 0.9$ & $10.5 \pm 0.8$ & $28.5 \pm 1.2$ & $4.3 \pm 0.2$ \\
龙 & & & & & \\
\hline
\end{tabular}

活性. 其中, 化合物 4c, 4g, 4i, 4j 体外抗肿瘤活性与阳 性对照物阿比特龙相当. 与其他人体癌细胞相比, 所测 化合物对人乳腺癌细胞株(MCF-7)具有更好的抗肿瘤活 性, 其 $\mathrm{IC}_{50}$ 值在 $3.0 \sim 25.5 \mu \mathrm{mol} / \mathrm{L}$. 从构效关系分析来 看, 噁唑环和噁唑 C-2 位置取代基的不同对化合物的 抗肿瘤活性影响较大: 通过在甾炔 3 的 17-位引入噁唑 环, 化合物的抗肿瘤活性明显增强; 增加烷基链的长度, 啞唑 2-位取代基由甲基(4a)、丙基(4b)到丁基(4c), 化合 物的抗肿瘤活性呈现明显的递增趋势; 将丙基(4b)取代 换成环丙烷基 $(\mathbf{4 g})$, 也有利于抗肿瘤活性的提高; 与 $\mathbf{4 b}$
相比, 化合物 $\mathbf{4 e}$ 抗肿瘤活性变化不是很明显. 我们也 研究了 2-位连芳基的情况, 2-苯基(4h)取代的抗肿瘤效 果远不如 2-吡啶基(4i)取代、对甲基芳基取代基 $(4 \mathbf{j})$ 和邻 溴芳基取代(4k). 噁唑 C-2 位置取代基对抗肿瘤活性的 影响以及其作用机理我们将进一步深入研究.

\section{2 结论}

以 4-雄烯二酮 $(1)$ 为原料, 用金催化甾炔法设计并 合成了一系列 17-(2',5'-二取代噁唑基)-雄甾-4,16-二烯3-䣶化合物 $\mathbf{4 a} \sim \mathbf{4 k}$ ，与其它合成 17-位杂环噁唑相比， 此合成方法具有反应路线短、产率高、反应条件温和等 优点. 体外抗肿瘤活性表明, 所测化合物对人乳腺癌细 胞株(MCF-7)具有更好的抗肿瘤活性, $\mathrm{IC}_{50}$ 值在 $3.0 \sim$ $25.5 \mu \mathrm{mol} / \mathrm{L}$, 且化合物 $\mathbf{4 c}, \mathbf{4 g}, \mathbf{4 i}, \mathbf{4 j}$ 抗肿瘤活性与阳性 对照物阿比特龙相当.

\section{3 实验部分}

\section{1 仪器与试剂}

反应过程使用薄层色谱板(TLC)监测; 快速柱色谱 使用柱层析硅胶(200 300 目); WPS-1 型数字熔点仪 (熔点未校正); ${ }^{1} \mathrm{H}$ NMR 和 ${ }^{13} \mathrm{C}$ NMR 用 Brucker ARX400 傅立叶变换核磁共振光谱仪测定 (TMS 为内标, $\mathrm{CDCl}_{3}$ 为 溶剂); IR 光谱表征使用 FD-5DX 红外光谱仪; HRMS 表 征使用 aZABHS 电喷雾电离质谱仪. 所用试剂如无特殊 说明, 均为市售分析纯, 除四氢呋喃需干燥纯化, 其它 试剂使用前均无进一步纯化.

实验所用人乳腺癌细胞 (MCF-7)、人肺癌细胞 (A549)、人肝癌细胞(Bel-7402)、人宫颈癌细胞(Hela)和 人前列腺癌细胞(PC-3M-1E8)由湘雅医院惠赠.

\section{2 实验方法}

\subsubsection{7-乙炔基-17-羟基-雄甾-4-烯-3-酮(2)的合成}

中间体 2 的制备参照文献[22]方法.

\subsubsection{7-乙炔基-雄留-4,16-二烯-3-酮(3)的合成}

中间体 3 的制备参照文献[23]方法.

3.2.3 17-(2',5'-二取代噁唑基)-雄甾-4,16-二烯-3-酮 $(4 a \sim 4 k)$ 的合成

将化合物 3 (0.30 mmol)溶于腈溶剂(3 mL)中, 加入 8-甲基喹啉氮氧(0.39 mmol, $62.0 \mathrm{mg}), \mathrm{Ph}_{3} \mathrm{PAuNTf}_{2}$ (11.1 $\mathrm{mg}, 0.015 \mathrm{mmol}), 60{ }^{\circ} \mathrm{C}$ 下反应, TLC 监测反应. 反应完 后减压旋去溶剂, 残留物用硅胶柱层析分离得到目标产 物 $4 \mathbf{a} \sim \mathbf{4 i}$, 对于化合物 $\mathbf{4 j}, 4 \mathbf{k}$ 的合成, 由于反应的腈是 固体, 无法作为反应的溶剂, 我们选用惰性溶剂氯苯 $(3$ $\mathrm{mL}$ )作溶剂, 加入 2 equiv. 固体腈, 8-甲基喹啉氮氧、金催 化剂以及反应温度都一样. 
17-(2'-甲基-5'-噁唑基)-雄甾-4,16-二烯-3-酮(4a): 淡黄色固体, 收率 70\%. m.p. 84 86 ${ }^{\circ} \mathrm{C} ;{ }^{1} \mathrm{H}$ NMR $(400$ $\left.\mathrm{MHz}, \mathrm{CDCl}_{3}\right) \delta: 6.83\left(\mathrm{~s}, 1 \mathrm{H}, 4{ }^{\prime}-\mathrm{H}\right), 6.06(\mathrm{t}, J=2.6 \mathrm{~Hz}, 1 \mathrm{H}$, 16-H), 5.75 (s, 1H, 4-H), 2.45 (s, 3H, $\left.\mathrm{CH}_{3}\right), 1.23$ (s, 3H, $\left.19-\mathrm{CH}_{3}\right), 0.98\left(\mathrm{~s}, 3 \mathrm{H}, 18-\mathrm{CH}_{3}\right) ;{ }^{13} \mathrm{C}$ NMR $(100 \mathrm{MHz}$, $\left.\mathrm{CDCl}_{3}\right) \delta: 199.50,170.93,160.65,148.02,141.47,126.17$, $123.99,121.57,56.05,53.92,46.33,38.67,35.51,34.96$, $33.90,33.85,32.71,31.70,31.54,20.86,17.18,16.36$, 13.96; IR (KBr) v: 2937.43, 1665.32, 1428.92, 1228.93 $\mathrm{cm}^{-1}$; HRMS calcd for $\mathrm{C}_{23} \mathrm{H}_{29} \mathrm{NO}_{2} 351.2198$, found 351.2193 .

17-(2'-丙基-5'-噁唑基)-雄甾-4,16-二烯-3-酮 (4b): 淡黄色固体, 收率 68\%. m.p. 87 89 ${ }^{\circ} \mathrm{C} ;{ }^{1} \mathrm{H}$ NMR $(400$ $\left.\mathrm{MHz}, \mathrm{CDCl}_{3}\right) \delta: 6.83\left(\mathrm{~s}, 1 \mathrm{H}, 4^{\prime}-\mathrm{H}\right), 6.03(\mathrm{t}, J=2.6 \mathrm{~Hz}, 1 \mathrm{H}$, 16-H), 5.73 (s, 1H, 4-H), 2.70 (t, $J=7.4 \mathrm{~Hz}, 2 \mathrm{H}, \mathrm{CH}_{2}$ ), 1.21 (s, 3H, 19- $\left.\mathrm{CH}_{3}\right), 0.98$ (s, 3H, 18- $\left.\mathrm{CH}_{3}\right), 0.98$ (t, $J=7.4$ $\left.\mathrm{Hz}, 3 \mathrm{H}, \mathrm{CH}_{3}\right) ;{ }^{13} \mathrm{C}$ NMR $\left(100 \mathrm{MHz}, \mathrm{CDCl}_{3}\right) \delta: 199.27$, $170.74,164.03,147.75,141.58,126,05,123.97,121.49$, $56.07,53.95,46.34,38.65,35.51,34.98,33.87,32.67$, $31.71,31.51,30.01,29.62,20.88,20.41,17.16,16.35$, 13.65; IR (KBr) v: 2935.72, 1662.64, 1436.96, 1228.65 $\mathrm{cm}^{-1}$; HRMS calcd for $\mathrm{C}_{25} \mathrm{H}_{33} \mathrm{NO}_{2}$ 379.2511, found 379.2507 .

17-(2'-丁基-5'-琴唑基)-雄甾-4,16-二烯-3-酮(4c): 淡黄色固体, 收率 65\%. m.p. 92 94 ${ }^{\circ} \mathrm{C} ;{ }^{1} \mathrm{H}$ NMR (400 $\left.\mathrm{MHz}, \mathrm{CDCl}_{3}\right) \delta: 6.82\left(\mathrm{~s}, 1 \mathrm{H}, 4^{\prime}-\mathrm{H}\right), 6.04$ (t, $J=2.2 \mathrm{~Hz}, 1 \mathrm{H}$, 16-H), 5.73 (s, 1H, 4-H), 2.73 (t, $J=7.2 \mathrm{~Hz}, 2 \mathrm{H}, \mathrm{CH}_{2}$ ), $1.22\left(\mathrm{~s}, 3 \mathrm{H}, 19-\mathrm{CH}_{3}\right), 0.98\left(\mathrm{~s}, 3 \mathrm{H}, 18-\mathrm{CH}_{3}\right), 0.92$ (t, $J=7.0$ $\left.\mathrm{Hz}, 3 \mathrm{H}, \mathrm{CH}_{3}\right) ;{ }^{13} \mathrm{C}$ NMR $\left(100 \mathrm{MHz}, \mathrm{CDCl}_{3}\right) \delta: 199.46$, $170.91,164.21,147.70,141.53,126,02,123.96,121.47$, $56.03,53.91,46.31,38.64,35.48,34.94,33.88,33.82$, $32.68,31.68,31.35,29.04,27.82,22.20,20.85,17.15$, 16.35, 13.67; IR (KBr) v: 2931.26, 1661.89, 1435.86, $1226.88 \mathrm{~cm}^{-1}$; HRMS calcd for $\mathrm{C}_{26} \mathrm{H}_{35} \mathrm{NO}_{2} 393.2668$, found 393.2662 .

17-(2'-戊腈基-5'-噁唑基)-雄甾-4,16-二烯-3-酮(4d): 黄色固体, 收率 $61 \%$. m.p. 97 98 ${ }^{\circ} \mathrm{C} ;{ }^{1} \mathrm{H}$ NMR (400 $\left.\mathrm{MHz}, \mathrm{CDCl}_{3}\right) \delta: 6.82\left(\mathrm{~s}, 1 \mathrm{H}, 4^{\prime}-\mathrm{H}\right), 6.05(\mathrm{t}, J=2.6 \mathrm{~Hz}, 1 \mathrm{H}$, 16-H), 5.72 (s, 1H, 4-H), 2.79 (t, $J=7.2 \mathrm{~Hz}, 2 \mathrm{H}, \mathrm{CH}_{2}$ ), $2.38\left(\mathrm{t}, J=7.0 \mathrm{~Hz}, 2 \mathrm{H}, \mathrm{CH}_{2} \mathrm{CN}\right), 1.21\left(\mathrm{~s}, 3 \mathrm{H}, 19-\mathrm{CH}_{3}\right) 0.97$ (s, 3H, $\left.18-\mathrm{CH}_{3}\right) ;{ }^{13} \mathrm{C}$ NMR $\left(100 \mathrm{MHz}, \mathrm{CDCl}_{3}\right) \delta: 199.35$, $170.80,162.68,148.09,141.29,126.59,123.92,121.43$, $119.23,56.00,53.87,46.30,38.61,35.45,34.90,33.84$, $33.78,32.63,31.64,31.51,27.15,25.78,24.63,20.81$, $17.12,16.84,16.33$; IR (KBr) $v: 2930.58,2232.24$,
1662.56, 1435.56, $1227.34 \mathrm{~cm}^{-1}$; HRMS calcd for $\mathrm{C}_{27} \mathrm{H}_{34} \mathrm{~N}_{2} \mathrm{O}_{2} 418.2620$, found 418.2616.

17-[2'-(1"-氯-丙基)-5-噁唑基]-雄甾-4,16-二烯-3-酮 (4e): 黄色固体, 收率 $63 \%$. m.p. $92 \sim 93{ }^{\circ} \mathrm{C} ;{ }^{1} \mathrm{H}$ NMR $\left(400 \mathrm{MHz}, \mathrm{CDCl}_{3}\right) \delta: 6.84\left(\mathrm{~s}, 1 \mathrm{H}, 4^{\prime}-\mathrm{H}\right), 6.07$ (t, $J=2.6$ $\mathrm{Hz}, 1 \mathrm{H}, 16-\mathrm{H}), 5.74$ (s, 1H, 4-H), $3.64(\mathrm{t}, J=6.2 \mathrm{~Hz}, 2 \mathrm{H}$, $\left.\mathrm{CH}_{2} \mathrm{Cl}\right), 2.93\left(\mathrm{t}, J=7.2 \mathrm{~Hz}, 2 \mathrm{H}, \mathrm{CH}_{2}\right), 1.22(\mathrm{~s}, 3 \mathrm{H}$, 19- $\left.\mathrm{CH}_{3}\right), 0.98\left(\mathrm{~s}, 3 \mathrm{H}, 18-\mathrm{CH}_{3}\right) ;{ }^{13} \mathrm{C}$ NMR $(100 \mathrm{MHz}$, $\left.\mathrm{CDCl}_{3}\right) \delta: 199.49,170.90,162.52,148.14,141.36,126.60$, $123.99,121.52,56.04,53.90,46.34,43.85,38.65,35.49$, $34.94,33.89,33.8332 .6831 .68,31.55,29.53,25.28$, $20.85,17.17,16.37$; IR (KBr) v: 2941.44, 1670.35, 1452.40, $1230.58 \mathrm{~cm}^{-1}$; HRMS calcd for $\mathrm{C}_{25} \mathrm{H}_{32} \mathrm{NO}_{2} \mathrm{Cl}$ 413.2122, found 413.2115.

17-(2'-乙烯基-5'-噁唑基)-雄甾-4,16-二烯-3-酮(4f): 黄色固体, 收率 $65 \%$. m.p. $78 \sim 79{ }^{\circ} \mathrm{C} ;{ }^{1} \mathrm{H}$ NMR $(400$ $\mathrm{MHz}, \mathrm{CDCl}_{3}$ ) $\delta: 6.99$ (s, 1H, 4'-H), 6.56 (dd, $J=11.2,11.2$ $\mathrm{Hz}, 1 \mathrm{H},=\mathrm{CH}), 6.17(\mathrm{t}, J=2.2 \mathrm{~Hz}, 1 \mathrm{H}, 16-\mathrm{H}), 6.12(\mathrm{~d}$, $\left.J=10.8 \mathrm{~Hz}, 1 \mathrm{H},=\mathrm{CH}_{2}\right), 5.75(\mathrm{~s}, 1 \mathrm{H}, 4-\mathrm{H}), 5.59(\mathrm{~d}, J=$ $\left.11.2 \mathrm{~Hz}, 1 \mathrm{H},=\mathrm{CH}_{2}\right), 1.23\left(\mathrm{~s}, 3 \mathrm{H}, 19-\mathrm{CH}_{3}\right), 1.00(\mathrm{~s}, 3 \mathrm{H}$, $\left.18-\mathrm{CH}_{3}\right) ;{ }^{13} \mathrm{C} \mathrm{NMR}\left(100 \mathrm{MHz}, \mathrm{CDCl}_{3}\right), \delta: 199.48,170.85$, $163.77,141.35,127.64,125.71,124.01,123.31,123.06$, $121.37,56.04,53.91,46.40,38.66,35.51,34.98,33.90$, $33.85,32.69,31.69,31.68,20.88,17.18,16.44$; IR (KBr) v: 2927.94, 1674.21, 1454.32, $1226.72 \mathrm{~cm}^{-1}$; HRMS calcd for $\mathrm{C}_{24} \mathrm{H}_{29} \mathrm{NO}_{2} 363.2198$, found 363.2193 .

17-(2'-环丙基-5'-噁坐基)-雄甾-4,16-二烯-3-酮(4g): 淡黄色固体, 收率 62\%. m.p. 127 129 ${ }^{\circ} \mathrm{C} ;{ }^{1} \mathrm{H}$ NMR $\left(400 \mathrm{MHz}, \mathrm{CDCl}_{3}\right) \delta: 6.68\left(\mathrm{~s}, 1 \mathrm{H}, 4{ }^{\prime}-\mathrm{H}\right), 6.00(\mathrm{t}, J=2.6$ $\mathrm{Hz}, 1 \mathrm{H}, 16-\mathrm{H}), 5.74(\mathrm{~s}, 1 \mathrm{H}, 4-\mathrm{H}), 1.22\left(\mathrm{~s}, 3 \mathrm{H}, 19-\mathrm{CH}_{3}\right)$, 0.97 (s, 3H, $\left.18-\mathrm{CH}_{3}\right) ;{ }^{13} \mathrm{C}$ NMR $\left(100 \mathrm{MHz}, \mathrm{CDCl}_{3}\right) \delta$ : $199.53,170.99,165.30,147.22,141.39,125.77,123.95$, $121.45,56.02,53.91,46.31,38.65,35.48,34.95,33.87$, $33.82,32.70,31.69,31.51,20.85,17.16,16.36,8.93,8.32$, 8.30; IR (KBr) v: 2939.51, 1674.21, 1450.47, 1226.72 $\mathrm{cm}^{-1}$; HRMS calcd for $\mathrm{C}_{25} \mathrm{H}_{31} \mathrm{NO}_{2}$ 377.2355, found 377.2350 .

17-(2'-苯基-5'-噁唑基)-雄甾-4,16-二烯-3-酮(4h): 淡黄色固体, 收率 68\%. m.p. $218 \sim 220{ }^{\circ} \mathrm{C} ;{ }^{1} \mathrm{H}$ NMR $\left(400 \mathrm{MHz}, \mathrm{CDCl}_{3}\right) \delta: 8.03(\mathrm{t}, J=4.0 \mathrm{~Hz}, 2 \mathrm{H}, \mathrm{Ar}-\mathrm{H})$, $7.44 \sim 7.46$ (m, 3H, Ar-H), 7.10 (s, 1H, 4'-H), 6.23 (t, $J=$ $2.2 \mathrm{~Hz}, 1 \mathrm{H}, 16-\mathrm{H}), 5.76$ (s, 1H, 4-H), 1.24 (s, 3H, 19- $\mathrm{CH}_{3}$ ), $1.04\left(\mathrm{~s}, 3 \mathrm{H}, 18-\mathrm{CH}_{3}\right) ;{ }^{13} \mathrm{C}$ NMR $\left(100 \mathrm{MHz}, \mathrm{CDCl}_{3}\right), \delta$ : $199.52,170.93,160.62,148.32,141.44,130.26,128.76$, $127.36,127.30,126.21,124.01,123.19,56.07,53.95$, 
$46.51,38.68,35.51,35.04,33.89,32.71,31.71,20.91$, 17.19, 16.48; IR (KBr) v: 2926.01, 1666.49, 1448.54, $1226.72 \mathrm{~cm}^{-1}$; HRMS calcd for $\mathrm{C}_{28} \mathrm{H}_{31} \mathrm{NO}_{2}$ 413.2355, found 413.2351 .

17-[2'-(2"-吡啶基)-5'-噁唑基]-雄甾-4,16-二烯-3-酮 (4i): 淡黄色固体，收率 55\%. m.p. $169 \sim 171{ }^{\circ} \mathrm{C} ;{ }^{1} \mathrm{H}$ NMR (400 MHz, $\left.\mathrm{CDCl}_{3}\right) \delta: 8.73$ (d, $\left.J=4.4 \mathrm{~Hz}, 1 \mathrm{H}, \mathrm{Ar}-\mathrm{H}\right)$, $8.10(\mathrm{~d}, J=8.0 \mathrm{~Hz}, 1 \mathrm{H}, \mathrm{Ar}-\mathrm{H}), 7.80(\mathrm{t}, J=7.8 \mathrm{~Hz}, 1 \mathrm{H}$, Ar-H), 7.34 (t, $J=6.0 \mathrm{~Hz}, 1 \mathrm{H}, \mathrm{Ar}-\mathrm{H}), 7.14\left(\mathrm{~s}, 1 \mathrm{H}, 4^{\prime}-\mathrm{H}\right)$, $6.38(\mathrm{t}, J=2.2 \mathrm{~Hz}, 1 \mathrm{H}, 16-\mathrm{H}), 5.76(\mathrm{~s}, 1 \mathrm{H}, 4-\mathrm{H}), 1.25$ (s, $\left.3 \mathrm{H}, 19-\mathrm{CH}_{3}\right), 1.04\left(\mathrm{~s}, 3 \mathrm{H}, 18-\mathrm{CH}_{3}\right) ;{ }^{13} \mathrm{C}$ NMR $(100 \mathrm{MHz}$, $\left.\mathrm{CDCl}_{3}\right) \delta: 199.41,170.80,159.52,150.07,146.15,141.14$, $136.85,128.88,126.21,124.40,124.06,123.62,121.96$, $56.10,53.98,46.56,38.70,35.57,35.07,33.95,33.92$, $32.72,31.74,29.69,20.94,17.23,16.49$; IR (KBr) $v$ : 2926.25, 1666.28, 1485.76, $1228.89 \mathrm{~cm}^{-1}$; HRMS calcd for $\mathrm{C}_{27} \mathrm{H}_{30} \mathrm{~N}_{2} \mathrm{O}_{2}$ 414.2307, found 414.2303.

17-[2'-(4"- 甲基苯基)-5-哑唑基]-雄甾-4,16-二烯-3酮 $(4 \mathbf{j})$ : 淡黄色固体，收率 $60 \%$. m.p. $238 \sim 240{ }^{\circ} \mathrm{C} ;{ }^{1} \mathrm{H}$ NMR (400 MHz, $\left.\mathrm{CDCl}_{3}\right) \delta: 7.91(\mathrm{~d}, J=8.4 \mathrm{~Hz}, 2 \mathrm{H}, \mathrm{Ar}-\mathrm{H})$, 7.24 (d, J=8.0 Hz, 2H, Ar-H), 7.05 (s, 1H, 4'-H), 6.19 (t, $J=2.6 \mathrm{~Hz}, 1 \mathrm{H}, 16-\mathrm{H}), 5.74$ (s, 1H, 4-H), 2.39 (s, 3H, $4^{\prime \prime}-\mathrm{CH}_{3}$ ), 1.22 (s, 3H, 19- $\mathrm{CH}_{3}$ ), 1.04 (s, 3H, $\left.18-\mathrm{CH}_{3}\right) ;{ }^{13} \mathrm{C}$ NMR $\left(100 \mathrm{MHz}, \mathrm{CDCl}_{3}\right) \delta: 199.44,170.87,160.81$, $147.93,141.45,140.49,129.43,126.92,126.12,124.65$, $123.97,123.13,56.00,53.88,46.43,38.63,35.46,34.98$, $33.88,33.82,32.67,31.66,31.64,21.49,20.86,17.14$, 16.44; IR (KBr) v: 2924.08, 1664.56, 1492.90, 1228.65 $\mathrm{cm}^{-1}$; HRMS calcd for $\mathrm{C}_{29} \mathrm{H}_{33} \mathrm{NO}_{2}$ 427.2511, found 427.2506.

17-[2'-(2"-溴苯基)-5'-噁唑基]-雄甾-4,16-二烯-3-酮 (4k): 淡黄色固体, 收率 48\%. m.p. 209 211 ${ }^{\circ} \mathrm{C} ;{ }^{1} \mathrm{H}$ NMR (400 MHz, $\left.\mathrm{CDCl}_{3}\right) \delta: 7.97$ (dd, $J=1.6,1.6 \mathrm{~Hz}, 1 \mathrm{H}$, Ar-H), 7.71 (dd, $J=0.8,0.8 \mathrm{~Hz}, 1 \mathrm{H}, \mathrm{Ar}-\mathrm{H}), 7.41$ (t, $J=7.6$ $\mathrm{Hz}, 1 \mathrm{H}, \mathrm{Ar}-\mathrm{H}), 7.29$ (dd, $J=1.6,1.6 \mathrm{~Hz}, 1 \mathrm{H}, \mathrm{ArH}), 7.15$ (s, 1H, 4'-H), 6.25 (t, $J=2.4 \mathrm{~Hz}, 1 \mathrm{H}, 16-\mathrm{H}), 5.76$ (s, 1H, $4-\mathrm{H}), 1.25\left(\mathrm{~s}, 3 \mathrm{H}, 19-\mathrm{CH}_{3}\right), 1.06\left(\mathrm{~s}, 3 \mathrm{H}, 18-\mathrm{CH}_{3}\right) ;{ }^{13} \mathrm{C}$ NMR $\left(100 \mathrm{MHz}, \mathrm{CDCl}_{3}\right) \delta: 199.38,170.78,159.02$, $148.85,141.44,134.65,131.12,130.99,128.22,128.13$, $127.36,124.06,123.12,120.73,56.17,54.01,46.50,38.71$, $35.57,35.03,33.95,33.93,32.72,31.76,29.68,20.95$, 17.23, 16.49; IR (KBr) v: 2926.08, 1668.54, 1456.55, $1209.76,840.98,753.26 \mathrm{~cm}^{-1}$; HRMS calcd for $\mathrm{C}_{28} \mathrm{H}_{30} \mathrm{Br}-$ $\mathrm{NO}_{2} 491.1460$, found 491.1454 .

\subsection{MTT 法体外抗肿瘤活性实验}

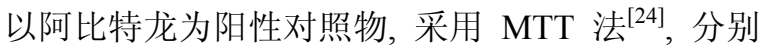
测试了化合物 3 和化合物 $\mathbf{4 a} \sim \mathbf{4 k}$ 对人乳腺癌细胞 $(\mathrm{MCF}-7) 、 人$ 肺癌细胞(A549)、人肝癌细胞(Bel-7402)、 人宫颈癌细胞(Hela)和人前列腺癌细胞(PC-3M-1E8)五 组细胞株的生长抑制情况.

称取 $10 \mu \mathrm{mol}$ 样品置于 $1.5 \mathrm{~mL}$ EP 管中, 用 DMSO 配制成 $10 \mu \mathrm{mol} / \mathrm{mL}$ 的溶液, 在 $4{ }^{\circ} \mathrm{C}$ 下保存, 根据实验 所需浓度用培养基稀释. 取肿瘤细胞株，用 RPMI-1640 培养液于 $\mathrm{CO}_{2}$ 孵箱中 $37{ }^{\circ} \mathrm{C}, 5 \% \mathrm{CO}_{2}$ 饱和湿度下培养, 细胞贴壁后用胰蛋白酶消化传代. 将处于对数生长期的 肿瘤细胞以 $5 \times 10^{4} / \mathrm{mL}$ 的密度接种于 96 孔培养板中, 每孔加 $100 \mu \mathrm{L}$. 培养 $48 \mathrm{~h}$ ，弃去培养基，每孔加入不同 浓度的被测化合物 $100 \mu \mathrm{L}$, 每个药设 5 个剂量组，每组 设 4 个平行孔. 另设空白组和对照组(以阿比特龙阳性 对照), 加药完毕后于 $\mathrm{CO}_{2}$ 孵箱中在 $37{ }^{\circ} \mathrm{C}, 5 \% \mathrm{CO}_{2}$ 饱和 湿度下培养 $48 \mathrm{~h}$, 上清液弃置, 然后每孔再加入 2.5 $\mathrm{mg} / \mathrm{mL}$ MTT 溶液 $40 \mu \mathrm{L}$, 继续培养 $4 \mathrm{~h}$, 上清液弃置, 每孔加入 $150 \mu \mathrm{L}$ DMSO, 振荡 $10 \mathrm{~min}(60$ 次 $/ \mathrm{min})$ 后, 用自动酶标仪于 $570 \mathrm{~nm}$ 波长处测定吸光度(OD)值, 取 各组平均值，重复实验 3 次. 按公式: 细胞增殖抑制率 $(\%)=($ 正常 OD 值一加药 OD 值 $) /$ 正常 OD 值 $\times 100 \%$ ，应 用 SPSS 软件计算半数抑制浓度.

\section{References}

[1] Salvador, J. A. R.; Carvalho, J. F. S.; Neves, M. A. C.; Silvestre, S M.; Leitao, A. J.; Silva, M. M. C.; Sae Melo, M. L. Nat. Prod. Rep. 2013, 30, 324.

[2] Guan, Y.-Y.; Zheng, D.; Yan, Z.; Wang, N.; Lei, P.-Y. Eur. J. Med. Chem. 2012, 51, 200

[3] Sica, D.; Musumeci, D. Steroids 2004, 69, 743.

[4] Dubey, R. K.; Oparil, S.; Imthurn, B.; Jackson, E. K. Cardiovasc. Res. 2002, 53, 688

[5] Wei, X.-L.; Shu, P.-H.; Liu, T.-T.; Xiang, M.; Zhang, J.-W.; Xue, Y.-B.; Luo, Z.-W.; Yao, G.-M..; Zhang, Y.-H. Chin. J. Org. Chem. 2013, 33, 1273 (in Chinese)

(魏夏兰，舒朋华，刘婷婷，向 明，张锦文，薛永波，罗增伟，姚 广民，张勇慧，有机化学, 2013, 33, 1273.)

[6] Mobley, J. A.; L'Esperance, J. O.; Wu, M.; Friel, C. J.; Hanson, R. H.; Ho, S.-M. Mol. Cancer Ther. 2004, 3, 587.

[7] Ling, Y.-Z.; Li, J.-S.; Liu, Y.; Kato, K.; Klus, G. T.; Brodie, A. J. Med. Chem. 1997, 40, 3297.

[8] Njar, V. C. O.; Kato, K.; Nnane, I. P.; Grigoryev, D. N.; Long, B. J.; Brodie, A. M. H. J. Med. Chem. 1998, 41, 902.

[9] Zhu, N.; Ling, Y.; Lei, X.; Handratta, V.; Brodie, A. M. H. Steroids 2003, 68, 603 .

[10] Bruttomesso, A. C.; Eiras, J.; Ramírez, J. A.; Galagovsky, L. R. Tetrahedron Lett. 2009, 50, 4022.

[11] Iványi, Z.; Wölfling, J.; Görbe, T.; Szécsi, M.; Wittmann, T.; Schneider, G. Steroids 2010, 75, 450.

[12] Gupta, R.; Pathak, D.; Jindal, D. P. Eur. J. Med. Chem. 1996, 31, 241.

[13] Sonego, J. M.; Cirigliano, A. M.; Cabrera, G. M.; Burton, G.; 
Veleiro, A. S. Steroids 2013, 78, 644.

[14] Kumar, A.; Ahmad, P.; Maurya, R. A.; Singh, A. B.; Srivastava, A. K. Eur. J. Med. Chem. 2009, 44, 109.

[15] Kumar, D.; Kumar, N. M.; Sundaree, S.; Johnson, E. O.; Sha, K. Eur. J. Med. Chem. 2010, 45, 1244.

[16] Brescia, M. R.; Rokosz, L. L.; Cole, A. G.; Stauffer, T. M.; Lehrach, J. M.; Auld, D. S. Bioorg. Med. Chem. Lett. 2007, 17, 1211.

[17] Freeman, F.; Chen, T.; van der Linden, J. B. Synthesis 1997, 861.

[18] Kondrat'eva, G. Y.; Aitzhanova, M. A.; Bogdanov, V. S.; Ivanova, Z. N. Bull. Acad. Sci. USSR. Div. Chem. Sci. 1978, $27,773$.

[19] He, W.-M.; Li, C.-Q.; Zhang, L.-M. J. Am. Chem. Soc. 2011, 133,
8482.

[20] Xie, L.-Y.; Wu, Y.-D.; Yi, W.-G., Zhu, L.; Xiang, J.-N.; He, W.-M. J. Org. Chem. 2013, 78, 9190.

[21] Xie, L.-Y.; Liang, Z.-W.; Yan, D.; He, W.-M.; Xiang, J.-N. Synlett 2013, 1809.

[22] Verkruijsse, H. D.; Graaf, W. D.; Brandsma, L. Synth. Commun. 1988, $18,131$.

[23] Sólyom, S.; Szilágyi, K.; Toldy, L. J. Prak. Chem. 1988, 330, 309.

[24] Liu, Z.-P.; Wei, W.-X.; Gan, C.-F.; Huang, Y.-M.; Liu, S.; Zhou, M.; Cui, J.-G. Chin. J. Org. Chem.. 2013, 33, 2551 (in Chinese). (刘志平, 韦万兴, 甘春芳, 黄燕敏, 刘盛, 周敏, 崔建国, 有机 化学, 2013, 33, 2551.) 